\section{Adult hypertropia: a guide to diagnostic evaluation based on review of 300 patients}

MA Tamhankar ${ }^{1}$, JH Kim² ${ }^{2}$ G-S Ying ${ }^{1}$ and NJ Volpe ${ }^{1}$

\begin{abstract}
Purpose To highlight the key clinical features of various aetiologies of adult hypertropia and to discuss the diagnostic approach towards evaluation of vertical double vision.

Methods This is a retrospective crosssectional study. A total of 300 consecutive patients with vertical diplopia were evaluated by a single neuro-ophthalmologist and strabismologist in a tertiary care setting from 2005-2008. The medical records of all patients with vertical diplopia coded with one of the following diagnoses; hypertropia, diplopia, thyroid eye disease, fourth nerve palsy, ocular myasthenia, congenital strabismus, and third nerve palsy were reviewed. The main outcome measures were determination of aetiologies of hypertropia.

Results Fourth nerve palsy and thyroid eye disease were the most common causes of vertical diplopia in our series and comprised more than $50 \%$ of patients. The other causes of vertical diplopia were ocular surgery, orbital fracture, neurosurgery, childhood strabismus, skew deviation, third nerve palsy, myasthenia gravis, and decompensated hyperphorias. Ocular motility deficits were seen in $33 \%$ of the cohort of whom thyroid eye disease comprised the largest group. Orbital ultrasonography was sensitive in detecting

\section{Introduction}

Vertical double vision is a common complaint in ophthalmology practice. Non-specialists may have concerns about evaluating patients presenting with vertical diplopia and identifying those who may require emergent management. In the evaluation of such patients, a careful history, and examination of extraocular muscle function is critical in arriving at an aetiologic diagnosis, ordering appropriate tests and avoiding unnecessary neuro-imaging. Older reports of the incidence and prevalence of hypertropias have mainly focused on aetiologies of childhood hypertropias. ${ }^{1-6}$ These studies constituted mass screening of variable paediatric populations by non-ophthalmologists for the prevalence of strabismus. Other studies have reviewed the incidence and aetiologies of third, fourth, and sixth cranial nerve paresis in the overall adult and paediatric population. ${ }^{7-10}$ Details of history and ophthalmic examination that is critical in establishing the aetiology of hypertropia are lacking from all of these studies. ${ }^{1-10}$ The primary objective of this study was to review the common aetiologies of vertical diplopia in adults and to establish a systematic approach towards evaluation of patients with hypertropias.
\end{abstract} thyroid orbitopathy.

Conclusion In the majority of patients, the aetiologies of hypertropias can be ascertained by history and careful ophthalmic examination alone. Fourth nerve palsy and thyroid eye disease were the most common causes of vertical diplopia in this series. Eye (2011) 25, 91-96; doi:10.1038/eye.2010.160; published online 5 November 2010

Keywords: hypertropia; vertical diplopia; fourth nerve palsy; thyroid eye disease

\section{Materials and methods}

This was a retrospective cross-sectional study. The medical records of 300 consecutive patients with hypertropia seen over a three-year period from 2005-2008 were reviewed retrospectively.

All patients were evaluated in an outpatient clinic by a single neuro-ophthalmologist and strabismologist with a surgical practice at a tertiary referral center. The majority of patients were referred by general ophthalmologists,
${ }^{1}$ Scheie Eye Institute, University of Pennsylvania, Philadelphia, PA, USA

${ }^{2}$ Wills Eye Institute, Thomas Jefferson University, Philadelphia, PA, USA

Correspondence: MA Tamhankar, University of Pennsylvania, Scheie Eye Institute, 51N, 39th Street, Philadelphia, PA 19104, USA

Tel: + 12156628042 ;

Fax: + 12152434694

E-mail: madhura.tamhankar @uphs.upenn.edu

Received: 7 June 2010 Accepted in revised form: 17 September 2010 Published online: 5 November 2010

The paper was presented as a poster at North American Neuro-Ophthalmology Society conference in March 2008, Orlando, FL, USA. This paper was presented at the Scheie Eye Institute Alumni conference in 2009 , Philadelphia, PA, USA. 
internists, and emergency room physicians with a referral diagnosis of 'double vision'. Charts that were coded with the diagnosis of hypertropia, diplopia, thyroid eye disease, fourth nerve palsy, ocular myasthenia, congenital strabismus, orbital fracture, third nerve palsy, and skew deviation were reviewed.

Pertinent data regarding nature of diplopia, past medical history of hypertension, diabetes, thyroid dysfunction, cerebrovascular accident, previous history of strabismus, ocular and orbital surgery, head and orbital trauma, and history of neurosurgical intervention was ascertained. All the patients had a complete eye examination including analyses of ocular motility. Presence of periocular swelling, measurement of proptosis by exophthalmometry, and associated eyelid signs such as retraction, lag, and ptosis was noted. Presence or absence of orbicularis oculi weakness was documented.

Evaluation of ocular motility was performed by testing ductions in all cardinal gazes. Alignment of the eyes was assessed by cover/uncover, alternate cover, and Maddox rod and the angle of deviation was measured with prisms, using alternate cover testing and Maddox rod when necessary, in primary position at $6 \mathrm{~m}$, at $33 \mathrm{~cm}$, and in eight gaze positions in selected patients. Presence of excyclotorsion was tested using double Maddox rod. Vertical fusional amplitudes were measured in patients with longstanding diplopia by measuring the amount of ocular misalignment the patient could tolerate (by addition of vertical prism by cover test) without experiencing diplopia. Data on neuro-imaging, orbital ultrasonography and pertinent laboratory results (complete blood count, erythrocyte sedimentation rate, and acetylcholine receptor antibody tests), final diagnosis and management was extracted and entered into an Access Database form for purpose of analyses. Approval from the University of Pennsylvania IRB was obtained. Descriptive data analyses were performed to describe the aetiologies of hypertropia, and their clinical features.

\section{Results}

Of the 300 patients in the study, 145 were males and 155 were females. The mean age of the cohort was 54.9 years (Standard Deviation (SD): 17.5 years) with a range between 13-87 years. Only seven patients were between the ages of 13-18 (2.3\%). The distribution of aetiologies of hypertropia is depicted in Figure 1. The onset of diplopia was less than 1 month in 33 patients, between 1-6 months in 85 patients and more than 6 months in 182 patients.

Congenital fourth nerve palsy and thyroid eye disease comprised $23.3 \%(70 / 300)$ and $21.3 \%(64 / 300)$ of the cohort, respectively, and were the two most common aetiologies seen in our series. In addition, 9\% (27/300)

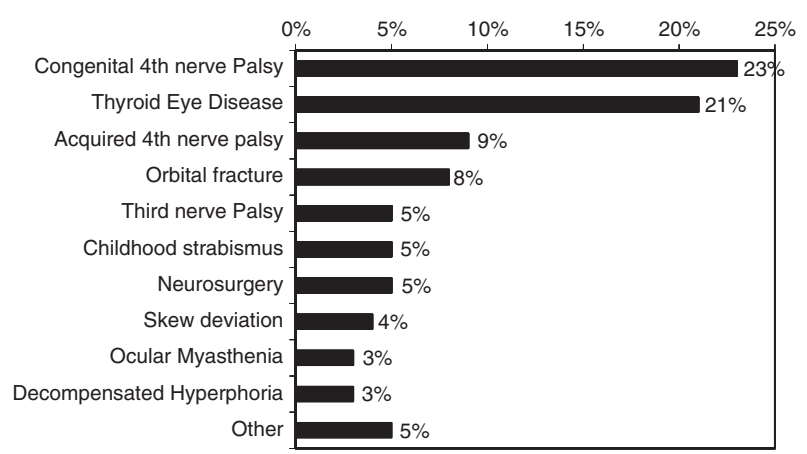

Figure 1 The various aetiologies of Hypertropia, $n=300$.

of the cohort had acquired fourth nerve palsy. Other aetiologies included orbital fracture and previous ocular surgery $8 \%(24 / 300)$ each, previous intracranial surgery $5 \%(15 / 300)$, previous strabismus $4.6 \%(14 / 300)$, skew deviation $4 \%(12 / 300)$, third nerve palsy $5 \%(15 / 300)$, ocular myasthenia gravis $3.3 \%$ (10/300), chronic hyperphorias 3\% (9/300), and miscellaneous causes $(5.3 \%, 16 / 300)$.

In the patients who presented with symptomatic hypertropia, the diagnosis of congenital fourth nerve palsy was established by a number of criteria based on history and clinical examination. A history of intermittent long-standing diplopia, head-tilt dating back to childhood as determined by history, examination, and old photographs were important considerations.

Examination features included presence of ipsilateral hypertropia that increased in contralateral gaze and ipsilateral head tilt (Parks three-step test), presence of ipsilateral inferior oblique muscle overaction (elevation of the eye in adduction), large fusional amplitudes (greater than five degrees) ${ }_{1}^{11}$ and absence of excyclotorsion. Figure 2 shows the frequency of occurrence of these features in our series $x$ of patients. In all, $30 \%$ of patients with congenital fourth nerve palsy had a sub-acute onset of double vision that is sometimes seen with decompensation of a pre-existing deviation. The history and examination features discussed above confirmed the presence of congenital fourth nerve palsy in these patients. Only one patient in the congenital fourth nerve palsy group (1.4\%) had a significant excyclotorsion as measured with a double Maddox rod test.

A total of 27 patients were diagnosed with acquired fourth nerve palsy secondary to trauma $(n=16)$, presumed vasculopathic aetiology $(n=9)$, and schwannomas of the fourth nerve $(n=2)$. Five of the 16 patients (four traumatic and one vasculopathic, 18.5\%) had significant excyclotorsion causing torsional diplopia and image tilt. Among these five patients, two were diagnosed with bilateral fourth nerve palsies based 


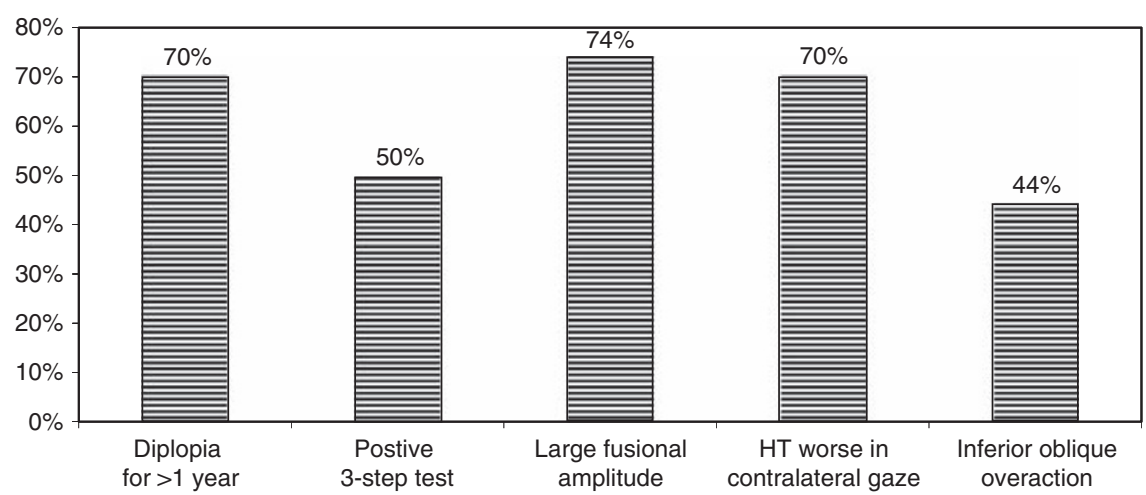

Figure 2 Key clinical features of congenital fourth nerve palsy.

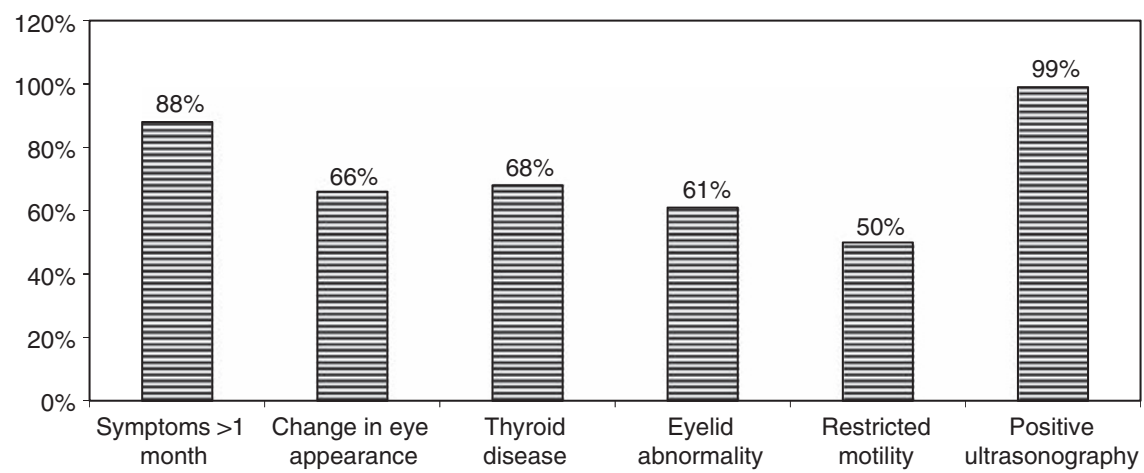

Figure 3 Key clinical features of thyroid eye disease.

on 10 degrees of excyclotorsion and hypertropia worsening in contralateral gaze. ${ }^{12}$ A positive three-step test was seen in 15/16 patients with traumatic and all patients with presumed vasculopathic fourth nerve palsy.

Thyroid eye disease was present in $21.3 \%$ (64/300) of the cohort and was characterized by proptosis, periocular swelling, and eyelid abnormalities such as eyelid retraction and/or eyelid lag (Figure 3). Previous history of systemic thyroid dysfunction was present in $68 \%$ whereas 32\% denied any thyroid dysfunction. Observed restriction of ocular motility was observed in more than 50\% (33/64) of patients. Ultrasonography showing enlarged extraocular muscles with high reflectivity was noted in 35 out of 36 patients (99\%) in whom it was performed. The remaining 28 patients were diagnosed based on CT/MRI findings of enlarged extraocular muscles. In 4/64 patients the diagnosis of thyroid orbitopathy was solely based upon ultrasound that revealed enlarged muscles with high reflectivity in the absence of any external signs of thyroid eye disease. These patients were labelled as having occult thyroid orbitopathy. ${ }^{13}$ One patient in this cohort was diagnosed with myasthenia gravis in conjunction with thyroid eye disease after presenting with new onset ptosis and was found to have positive acetylcholine receptor antibody test.
Among the cohort 8\% (24/300) reported vertical diplopia immediately after ocular surgery that included placement of scleral buckle $(n=8)$ and retrobulbar anaesthetic injection $(n=9)$. Other causes included tube shunt procedure, macular translocation surgery, orbital implant, retinal detachment surgery without scleral buckle placement, and strabismus surgery. The major causes of diplopia in this group were mechanical restriction from implanted hardware and myotoxicity of retrobulbar anaesthetic agent leading to paretic or restrictive forms of hypertropia.

A total of 14 patients (4.6\%) reported a past history of congenital or childhood strabismus with 11/14 having had previous eye-muscle surgery. The motility patterns were hypertropia with various combinations of eso- $(n=5)$ and exodeviations $(n=3)$ and one patient each with dissociated vertical deviation, double-elevator palsy, superior oblique overaction, and congenital nystagmus. The fewer number of patients with hypertropia occurring in conjunction with horizontal deviations, was likely because of the consequence of childhood strabismus that precluded binocular fusion, which led to patients not having symptomatic vertical diplopia. As the search criteria for this study was a referral diagnosis of vertical diplopia, patients with asymptomatic hyperdeviation seen in conjunction 
with exo- and eso-deviations were excluded from this analyses.

A total of 15 patients (5\%) of the cohort had postoperative diplopia from a previous neuro-surgical procedure and are grouped separately as their diplopia was directly linked to the procedure performed. The aetiologies included fourth nerve palsy $(n=8)$, skew deviation $(n=4)$, and third nerve palsy $(n=3)$.

Among the cohort 4\% (12/300) had skew deviation. An acute onset of diplopia, an older age group in this cohort (average age 68), a comitant nature of vertical deviation, absence of torsion and presence of associated brain-stem signs such as inter-nuclear ophthalmoplegia $(n=3)$ helped to differentiate skew deviation from fourth nerve palsy. In 10 patients the skew deviation was secondary to cerebrovascular accident, whereas one patient each had brain stem encephalitis and head trauma that caused a skew.

Among the cohort 5\% (15/300) had third nerve palsy and presented with ptosis, hypertropia with exotropia, and ocular motility deficits. In all, $40 \%$ of patients exhibited hypertropia that switched from upgaze to downgaze. The aetiologies were vasculopathic $(n=4)$, head trauma $(n=4)$, and one patient each with cavernous sinus meningioma, posterior communicating artery aneurysm, radiation, midbrain ischaemia, orbital lymphoma, and two patients with previous history of intracranial aneurysms complicated by subarachnoid haemorrhage. The patients with cavernous sinus meningioma and posterior communicating artery aneurysm had aberrant regeneration of third nerve characterized by elevation of the upper eyelid in adduction along with other features of third nerve palsy. Pupillary involvement from third nerve palsy was observed in 11/15 patients.

Ocular myasthenia gravis was diagnosed in 10 patients. Fatigability of affected extraocular muscles including levator palpebrae superioris, presence of variable ptosis $(9 / 10)$, presence of orbicularis oculi weakness $(5 / 10)$, and a positive acetylcholine receptor antibody (5/10) test helped to make the diagnosis.

Nine patients (3\%) were labelled as having chronic decompensated hypertropia following a history that suggested long-standing, intermittent nature of diplopia in whom the alternative aetiologies such as myasthenia gravis, fourth nerve palsy seemed less likely based on history and pattern of ocular misalignment.

Intracranial and intraorbital lesions causing hypertropia had a low prevalence in our series (2\%, 6/300 patients). Two patients with orbital masses (lymphoma, malignant tumour) presented with proptosis, restricted ocular motility, peri-ocular numbness, eye pain, and vision loss that prompted neuro-imaging and detection of orbital mass.
Additionally, two patients had chronic fourth nerve palsy from schwannomas that was detected with neuroimaging that was ordered because of non-resolution of the palsy. The patient with cavernous sinus meningioma had aberrant regeneration of the third nerve that prompted neuro-imaging and one patient had third ventricular tumour as a cause of episodic hyperdeviation.

Miscellaneous causes of hypertropias included Brown syndrome (2), Parkinson's disease (3), Carotid-cavernous fistula (2), superior oblique myokymia (2), orbital mass (2), and one each with carcinomatous meningitis, lyme disease, traumatic aphakia, superior oblique overaction, and third ventricular tumour.

In our series, observable limitation of eye movements was detected in a third of patients (108/300). The most common aetiology of obvious restricted extraocular motility was thyroid eye disease that was present in $33 / 108$. The other diagnoses of observed motility deficits were myasthenia gravis, third nerve palsy, orbital fracture, intraocular surgery, neurosurgery, and previous strabismus. The patients who had normal ocular ductions $(64 \%)$ were the fourth nerve palsy, skew deviation, and decompensated phoria sub-groups.

\section{Discussion}

Epidemiological studies on incidence and/or prevalence of vertical strabismus are few and primarily involve non-population-based screening of paediatric population ${ }^{1-6}$ performed by non-specialists and lacking details of examination and aetiologic diagnoses. One recent study found fourth nerve palsies to be the most common aetiology of hypertropia in 42 children (31\%). ${ }^{6}$ Our series concurs with this smaller paediatric series ${ }^{6}$ in which fourth nerve palsy was the most common aetiology representing 35\% of the cohort. The causes of fourth nerve palsy in our series is comparable with Von Noorden's series of 270 patients in which congenital and traumatic causes were the most common. ${ }^{14}$

Thyroid eye disease was the second most common cause for vertical diplopia, which was primarily diagnosed on the basis of associated external-eye signs such as proptosis, eyelid retraction, and lag. Ancillary modalities such as CT, MRI, and ultrasonography were useful in revealing enlargement of the extraocular muscles and high reflectivity on A scan, in the absence of external signs of thyroid dysfunction. ${ }^{15-18}$ The possibility of occult thyroid eye disease as a cause of unexplained ocular misalignment must be considered in patients in whom history or examination is not suggestive of previous thyroid dysfunction. ${ }^{13}$

In such patients ocular ultrasonography is a useful diagnostic tool to look for thickening and high reflectivity 
of extraocular muscles. In patients presenting with vertical misalignment observable limitation of ocular ductions is not always obvious during examination. It is not uncommon in a tertiary referral-based practice such as ours to see patients who complain of vertical diplopia and have no obvious motility deficits, as these patients are more challenging for the general ophthalmologist to diagnose. In such situations, tests to look for ocular misalignment such as cover/uncover, alternate cover, and Maddox rod testing should be used to uncover subtle deviations. Observed limitation of ocular motility is most often seen in hypertropia due to thyroid eye disease.

Vertical diplopia occurring after intraocular surgery is well recognized and multifactorial in its aetiology and usually presents acutely after cataract surgery.

Post-cataract surgery diplopia was present in $8 \%$ of patients in one study looking at aetiologies of adult onset strabismus. ${ }^{19}$ Decompensation of previously existing phoria, previous strabismus, myotoxicity of anaesthetic agent injected into the retrobulbar space, or restriction of extraocular muscle from scleral buckle or glaucoma shunt device are some proposed mechanisms of diplopia in this category. ${ }^{20,21}$ With myotoxicity from anaesthetic agent, the diplopia occurs acutely after surgery and the pattern of strabismus may change over time from paresis to restriction.

Skew-deviation results due to abnormalities in the vestibular periphery, brainstem, or cerebellum..$^{22,23}$
In such patients, presence of other brainstem findings such as nystagmus, inter-nuclear ophthalmoplegia, and saccadic and pursuit dysmetria in association with acute-onset hypertropia should raise the suspicion of skew deviation.

Our series provides data on the aetiologies of various types of adult hypertropias based on clinical history and examination. We did not encounter any other comparable studies of vertical diplopia in adults in our English language search in Pubmed that have systematically evaluated frequencies of aetiologies of adult hypertropias. In an older study looking at third, fourth and sixth nerve palsies occurring in isolation or in combination with neurologic symptoms in more than 4000 patients, almost $25 \%$ of patients had cranial nerve palsy of undetermined aetiology. ${ }^{7}$ Detailed ophthalmic evaluation and neuro-imaging data is lacking from this study. In another study of 104 adults undergoing strabismus surgery, $17 \%$ of patients had hypertropias due to previous strabismus (29\%) followed by third, fourth and sixth nerve palsies seen in $23 \%$ of patients. ${ }^{19}$

Our study is unique in that it highlights the most common aetiologies of vertical diplopia, as well as outlines a systematic approach towards a clinical evaluation including history, analyses of ocular motility, and alignment, laboratory, and neuro-imaging data to establish the diagnosis of hypertropia (Figure 4). MRI and orbital ultrasonography are useful modalities that

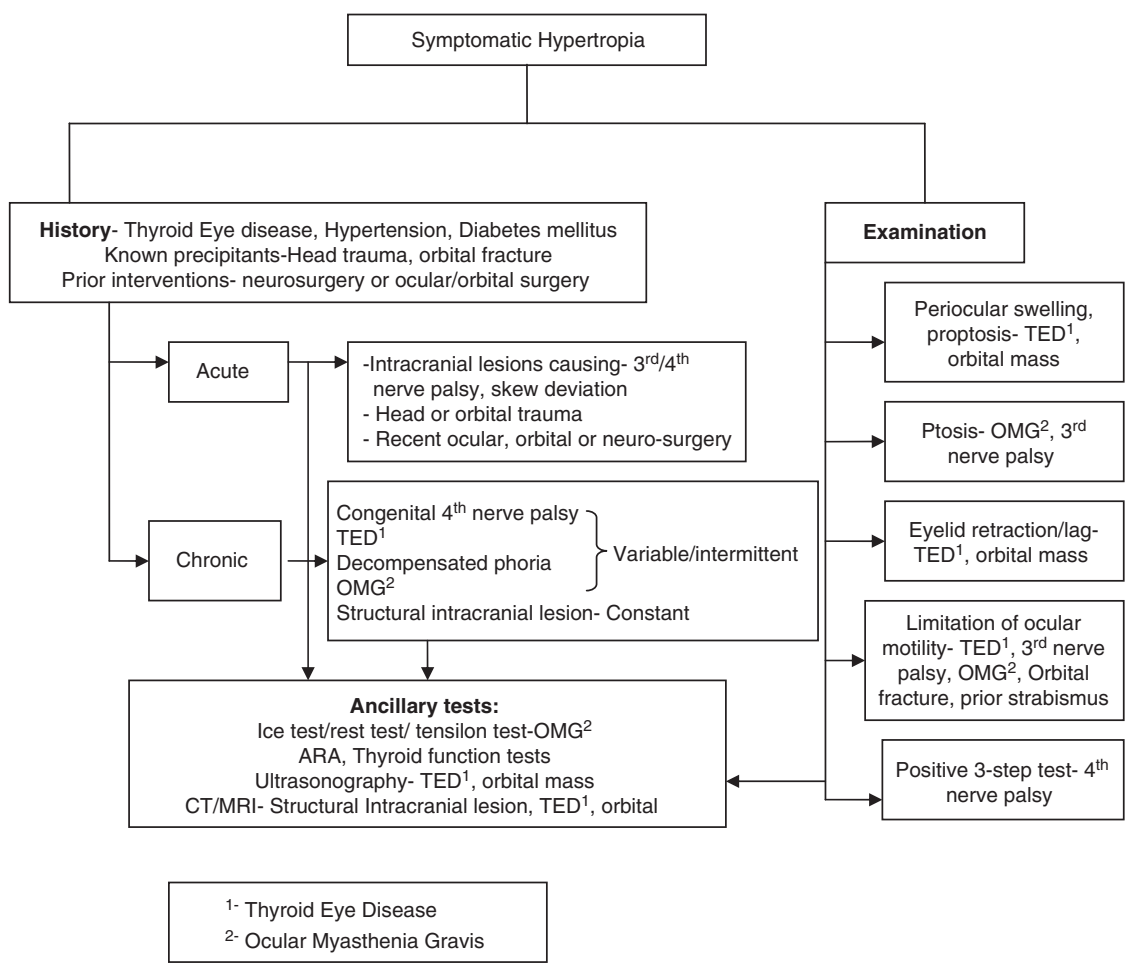

Figure 4 A flow chart highlighting the most common aetiologies and their evaluation. 
can help to secure the diagnosis in many patients, in whom the aetiology would have been 'indeterminate' as in studies in older literature. ${ }^{7}$ Although the clinical features of the various causes of vertical diplopia have been described before, a systematic evaluation of a large number of patients presenting with vertical diplopia is unique and will serve as a useful guide to the non-specialist when evaluating such patients.

Limitations of our study were that patients in our series were seen as out patients that incurred a referral bias favoring a higher prevalence for chronic diseases (thyroid eye disease and congenital fourth nerve palsy) and preexisting conditions such as ocular surgery, neurosurgery, orbital trauma, and congenital strabismus. Moreover, there were patients in this series who had previous neuro-imaging performed before they were seen by the neuro-ophthalmologist.

Our study provides data on the systematic approach to evaluating patients with hypertropias. We believe that this information will be valuable to the general ophthalmologists in making accurate diagnosis, creating critical management pathways, optimizing use of health care resources, and making appropriate referrals to specialists.

\section{Summary}

\section{What was known before}

- Clinical features of different etiologies of hypertropias has been well described in literature.

What this study adds

- This is a unique study that highlights the various etiologies of adult hypertropias in a large number of patients with emphasis on detailed clinical evaluation. This information will benefit the comprehensive ophthalmologist and guide him towards systematic evaluation of adult hypertropias and provide critical management pathways.

\section{Conflict of interest}

The authors declare no conflict of interest.

\section{Acknowledgements}

This study was supported by Research to prevent blindness.

\section{References}

1 Kornder LD, Nursey JN, Pratt-Johnson JA, Beattie A. Detection of manifest strabismus in young children. I. A prospective study. Am J Ophthalmol 1974; 77: 207-210.
2 Friedman Z, Neumann E, Hyams SW, Peleg B. Ophthalmic screening of 38000 children, age 1-2 1/2 years, in child welfare clinics. J Pediatr Ophthalmol Strabismus 1980; 17: 261-267.

3 Nordloew W. Squint - the frequency of onset at different ages, and the incidence of some associated defects in a Swedish population. Acta Ophthalmol (Copenh) 1964; 42: 1015-1037.

4 Kornder LD, Nursey JN, Pratt-Johnson JA, Beattie A Detection of manifest strabismus in young children. A retrospective study. Am J Ophthalmol 1974; 77: 211-214.

5 Frandsen AD. Some results from a clinical-statistical survey on strabismus among copenhagen children. Acta Ophthalmol (Copenh) 1958; 36(3): 488-498.

6 Tollefson MM, Mohney BG, Diehl NN, Burke JP. Incidence and types of childhood hypertropia: a population-based study. Ophthalmology 2006; 113: 1142-1145.

7 Richards BW, Jones Jr FR, Younge BR. Causes and prognosis in 4278 cases of paralysis of the oculomotor, trochlear, and abducens cranial nerves. Am J Ophthalmol 1992; 113: 489-496.

8 Rucker CW. Paralysis of the third, fourth and sixth cranial nerves. Am J Ophthalmol 1958; 46: 787-794.

9 Rucker CW. The causes of paralysis of the third, fourth and sixth cranial nerves. Am J Ophthalmol 1966; 61: 1293-1298.

10 Rush JA, Younge BR. Paralysis of cranial nerves III, IV, and VI. Cause and prognosis in 1000 cases. Arch Ophthalmol 1981; 99: 76-79.

11 Parks MM. Isolated cyclovertical palsy. Arch Ophthalmol 1958; 60(6): 1027-1035.

12 Pollard ZF. Classification and treatment of bilateral superior oblique palsy. Ann Ophthalmol 1985; 17: 127-131.

13 Volpe NJ, Sbarbaro JA, Gendron-Livingston K, Galetta SL, Liu GT, Balcer LJ. Occult thyroid eye disease in patients with unexplained ocular misalignment identified by standardized orbital echography. Am J Ophthalmol 2006; 142: 75-81.

14 Von Noorden GK, Murray E, Wong SY. Superior oblique paralysis. A review of 270 cases. Arch Ophthalmol 1986; 104: 1771-1776.

15 Trokel SL, Hilal SK. Recognition and differential diagnosis of enlarged extraocular muscles in computed tomography. Am J Ophthalmol 1979; 87: 503-512.

16 Char DH. Thyroid eye Disease. 3rd edn. ButterworthHeinemann: Boston, 1997.

17 Bryne SF, Green RL. Ultrasound of the Eye and Orbit. Mosby: St Louis, Missouri, 1992 pp 353-392.

18 Given-Wilson R, Pope RM, Michell MJ, Cannon R, McGregor AM. The use of real-time orbital ultrasound in Graves' ophthalmopathy: a comparison with computed tomography. Br J Radiol 1989; 62: 705-709.

19 Magramm I, Schlossman A. Strabismus in patients over the age of 60 years. J Pediatr Ophthalmol Strabismus 1991; 28: 28-31.

20 Costa PG, Debert I, Passos LB, Polati M. Persistent diplopia and strabismus after cataract surgery under local anesthesia. Binocul Vis Strabismus Q 2006; 21(3): 155-158.

21 Capó H. Diplopia after cataract surgery. Semin Ophthalmol 1999; 14(2): 62-64.

22 Keane JR. Ocular skew deviation: analysis of 100 cases. Arch Neurol 1975; 32: 185-190.

23 Brandt T, Dieterich M. Skew deviation with ocular torsion: a vestibular brainstem sign of topographic diagnostic value. Ann Neurol 1993; 33: 528-534. 